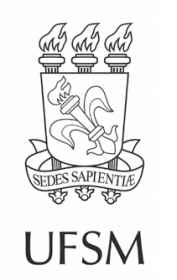

\title{
Artigos
}

\section{Efeito da extração alcalina na redução do número Kappa no branqueamento ECF}

\author{
Effect of alkaline extraction in reducing the Kappa number in ECF \\ bleaching
}

\section{Iliane Rodrigues de Oliveira ${ }^{\circ}$, , Gustavo Ventorim ${ }^{\circ}$, Ana Paula Almeida Ferraz "๑}

\author{
' Universidade Estadual Paulista "Júlio de Mesquita Filho", Itapeva, SP, Brasil \\ " Universidade Estadual Paulista "Júlio de Mesquita Filho"; Anhanguera Educacional, Itapeva, SP, Brasil
}

\section{RESUMO}

O estágio de hidrólise ácida $\left(\mathrm{A}_{\mathrm{HT}}\right)$, usado em sequências de branqueamento de polpa, tem como objetivo principal a degradação dos ácidos hexenurônicos, que estando presentes na polpa podem afetar sua alvura, reversão de alvura, entre outros. Apesar do benefício da utilização do estágio de hidrólise ácida, o pH desta etapa não é o mais adequado para a degradação da lignina, pois esta é mais reativa em meio alcalino. Portanto, o presente trabalho buscou estudar uma melhoria no processo de branqueamento pela adição de um estágio de extração alcalina após o estágio $A_{H^{\prime}}$ obtendo o melhor aproveitamento possível do estágio de dióxido cloro. Para este estudo foi utilizada polpa Kraft de eucalipto prédeslignificada com oxigênio. Diferentes sequências de branqueamento ECF (Elemental Chlorine Free) foram realizadas na polpa, sendo elas: $D_{0}(E+P) D_{1}, A_{H T} D_{0}(E+P) D_{1}, A_{H T} / E D_{0}(E+P) D_{1}$ e $A_{H T} E D_{0}(E+P) D_{1}$. Os resultados encontrados com a inclusão do estágio de extração alcalina simples após a hidrólise ácida apontam um efeito positivo na sequência de branqueamento, havendo uma significativa redução do consumo de dióxido de cloro. A inclusão da extração alcalina simples também possibilitou a redução do número Kappa e aumento na alvura, com uma redução de 0,3 pontos na média do número Kappa e aumento de 1,3\% ISO na alvura, analisados após a extração alcalina com peróxido de hidrogênio da sequência $O A_{H T} / E D_{0}(E+P) D_{1}$, se comparada à sequência sem extração alcalina simples.

Palavras-chave: Eucalipto; Hidrólise ácida; Branqueamento 


\section{ABSTRACT}

The acid hydrolysis stage $\left(\mathrm{A}_{\mathrm{HT}}\right)$ used in bleaching sequences of pulp, has as its main objective, the degradation of hexenuronic acids which are present in the pulp and can affect its brightness, reversal of brightness, among others. Despite the benefit of using the acid hydrolysis stage, the $\mathrm{pH}$ of this stage is not the most adequate, but when it comes to the degradation of lignin, it is more reactive in alkaline medium. Therefore, the present work sought to study an improvement in the bleaching process by the addition of an alkaline extraction stage after $A_{H T}$ stage, obtaining the best possible use of the chlorine dioxide stage. In this study, oxygen-delignifiedKraft pulp from eucalyptus was used. Different ECF (Elemental Chlorine Free) bleaching sequences were performed in the pulp: $D_{0}(E+P) D_{1}$, $\mathrm{A}_{\mathrm{HT}} \mathrm{D}_{0}(\mathrm{E}+\mathrm{P}) \mathrm{D}_{1}, \mathrm{~A}_{\mathrm{HT}} / \mathrm{ED}_{0}(\mathrm{E}+\mathrm{P}) \mathrm{D}_{1}$ and $\mathrm{A}_{\mathrm{HT}} \mathrm{ED}_{0}(\mathrm{E}+\mathrm{P}) \mathrm{D}_{1}$. As a result, the inclusion of a simple alkaline stage after the acid hydrolysis indicate a positive effect on the bleaching sequence, with a significant reduction in the consumption of chlorine dioxide. The inclusion of simple alkaline extraction also allowed the reduction of thekappa number and increased brightness, with a reduction of 0.3 points in the average Kappa number and a 1.3\% ISO increase in brightness, analyzed after alkaline extraction with hydrogen peroxide of the sequence $A_{H T} / E D_{0}(E+P) D_{1}$ when compared to the one without simple alkaline extraction.

Keywords: Eucalyptus; Acid hydrolysis; Bleaching

\section{INTRODUÇÃO}

Durante o branqueamento da polpa celulósica visa-se principalmente a eliminação da lignina residual para o alcance de um grau de alvura mais elevado, com a qualidade final da polpa dentro dos parâmetros estabelecidos. A lignina é um dos principais agentes cromóforos presentes na polpa. Além da lignina, os ácidos hexenurônicos (HexA) são também significativos(CADENA et al., 2010).

Os grupos HexA são significativos devido ao seu papel no processo de branqueamento e sua influência nas propriedades finais da polpa. Podendo aumentar o consumo de reagentes de branqueamento, o número Kappa e a reversão de alvura, através da retenção de íons metálicos (CADENA et al., 2010).

Os HexA são formados a partir da reação do álcali com ácido 4-O-metilglucurônico, presente nas xilanas, que são as hemiceluloses mais importantes da madeira de eucalipto. Como são gerados durante a polpação Kraft, sua quantidade na polpa é influenciada pelo tipo de madeira e pelas condições do processo, como o álcali e a temperatura empregada (VUORINEN et al., 1999; CADENA et al., 2011). 
Para polpas não branqueadas a presença de ácidos hexenurônicos não é de grande importância, entretanto quando presentes em polpas a serem branqueadas, afetam significativamente o branqueamento. Durante essa etapa, uma fração dos reagentes químicos que promovem ataque eletrofílico em meio ácido, como o dióxido de cloro, é consumido pelos ácidos hexenurônicos (REIS, 2013; COLODETTE; GOMES, 2015). Esses ácidos podem ser removidos seletivamente, com o auxílio de uma hidrólise ácida em alta temperatura $\left(\mathrm{A}_{\mathrm{HT}}\right)$. O uso de um estágio de hidrólise ácida antes do branqueamento pode reduzir o número Kappa em até 50\%, resultando em redução no consumo de reagentes de branqueamento. Esse estágio de hidrólise ácida pode ser utilizado tanto em sequências ECF quanto TCF. Pois, sob condições ácidas, os ácidos hexenurônicos são hidrolisados muito mais rapidamente que outras estruturas de carboidratos e ao mesmo tempo, o número Kappa diminui (VUORINEN et al.,1999).

Segundo Zhang et al. (2019), a remoção dos ácidos hexenurônicos implica em uma redução do número Kappa e consequentemente diminui a reversão de alvura. Em seus estudos, os autores observaram que o aumento da temperatura no estágio de hidrólise ácida acarreta decréscimo no teor de ácidos hexenurônicos na polpa, mantendo-se as outras condições constantes. Pois, com a redução doHexA residual, o número de reversão também diminuiu. De acordo com Cadena et al., (2011), a redução do conteúdo de HexA com o aumento da temperatura é devido a fragmentação das ligações dos complexos lignina-HexA-xilana.

Entretanto, apesar das condições da hidrólise ácida proporcionarem uma boa eficiência na remoção dos ácidos hexenurônicos, elas não são adequadas para a remoção ou degradação da lignina.

O branqueamento é realizado segundo a combinação de diversos estágios, utilizando diferentes reagentes para obter-se uma alvura mais estável e uma polpa com melhores características físico-mecânicas. É comum o uso da alternância de reagentes entre oxidantes e redutores, incluindo uma lavagem entre os mesmos (DENCE; REEVE, 1996; VENTORIM; COLODETTE; EIRAS, 2009). 
Portanto, procurou-se por meio deste estudo aperfeiçoar o processo de branqueamento, fazendo uma extração alcalina simples (E) após o estágio de hidrólise ácida, para que assim, além da remoção dos ácidos hexenurônicos alcançada pela hidrólise ácida, ocorra uma maior reatividade da lignina frente ao estágio de dióxido de cloro $\left(D_{0}\right)$. Assim, o objetivo deste trabalho foi estudar o decréscimo do número Kappa após a extração alcalina simples, e também avaliar a importância da hidrólise ácida numa sequência contendo duas diferentes modificações envolvendo os estágios $A_{H T}$ e $D_{0}$.

\section{MATERIAIS E MÉTODOS}

\subsection{Material}

Como matéria-prima para este trabalho foi utilizada polpa celulósica prédeslignificada com oxigênio proveniente de híbrido de Eucalyptus urophylla x Eucalyptus grandis.

\subsection{Método}

Foram utilizadas neste estudo as seguintes sequências de branqueamento ECF: $D_{0}(E+P) D_{1}, A_{H T} D_{0}(E+P) D_{1}, A_{H T} / E D_{0}(E+P) D_{1}$ e $A_{H T} E D_{0}(E+P) D_{1}$, onde: $A_{H T}$ = estágio de hidrólise ácida em alta temperatura; $D_{0}=$ primeiro estágio de dioxidação; $A_{H T} / E$ = estágio de hidrólise ácida em alta temperatura seguida por uma extração simples sem lavagem intermediária; $E$ = estágio de extração alcalina simples; $(E+P)$ = estágio de extração alcalina com peróxido de hidrogênio; $\mathrm{D}_{1}=$ segundo estágio de dioxidação.Todas as sequências de branqueamento foram realizadas em três repetições, cujas condições gerais de cada estágio estão descritas na Tabela 1.

Os estágios foram efetuados em sacos de polietileno com amostras representativas de polpa. O licor de branqueamento foi adicionado à polpa em temperatura ambiente. $\mathrm{O}$ requerimento de $\mathrm{NaOH}$ ou $\mathrm{H}_{2} \mathrm{SO}_{4}$ para controle de $\mathrm{pH}$ foi 
realizado em estudo prévio com miniamostras de polpas. Terminada a reação, foram extraídas amostras de licor residual das polpas para análises de pH e residual (quando aplicável). A polpa foi lavada com o equivalente a $9 \mathrm{~mL}$ de água por grama de polpa absolutamente seca.

Tabela 1 - Condições gerais dos estágios de branqueamento

\begin{tabular}{lcccccc}
\hline \multirow{2}{*}{ Condições } & \multicolumn{6}{c}{ Estágio de Branqueamento } \\
\cline { 2 - 7 } & $\mathbf{A}_{\mathrm{HT}}$ & $\mathbf{A}_{\mathrm{HT}} / \mathbf{E}$ & $\mathbf{D}_{\mathbf{0}}$ & $\mathbf{E}+\mathbf{P}$ & $\mathbf{E}$ & $\mathbf{D}_{\mathbf{1}}$ \\
\hline Consistência, $\%$ & 10 & 10 & 10 & 10 & 10 & 10 \\
Temperatura, ${ }^{\circ} \mathrm{C}$ & 95 & $95 / 70$ & 60 & 70 & 70 & 70 \\
Tempo, min. & 120 & $120 / 15$ & 30 & 60 & 15 & 180 \\
$\mathrm{H}_{2} \mathrm{O}_{2}, \mathrm{~kg} / \mathrm{t}$ & - & - & - & 3 & - & - \\
$\mathrm{NaOH}, \mathrm{kg} / \mathrm{t}$ & - & 10 & - & 10 & 10 & - \\
$\mathrm{H}_{2} \mathrm{SO}_{4}, \mathrm{~kg} / \mathrm{t}$ & 5 & 5 & $*$ & - & - & - \\
$\mathrm{ClO}_{2} \mathrm{C} / \mathrm{Cl}{ }_{2}, \mathrm{~kg} / \mathrm{t}$ & - & - & $*$ & - & - & $*$ \\
\hline
\end{tabular}

Fonte: Autores (2020)

Em que: $A_{H T}=$ hidrólise ácida em alta temperatura; $D_{0}=$ primeiro estágio de dioxidação; $A_{H T} / E=$ estágio de hidrólise ácida em alta temperatura seguida por uma extração simples sem lavagem intermediária; $E$ = estágio de extração alcalina simples; $(E+P)$ = estágio de extração alcalina com peróxido de hidrogênio; $\mathrm{D}_{1}=$ segundo estágio de dioxidação; * = Dosagem variável.

\subsection{Métodos de análise da polpa}

Os métodos de análises das polpas utilizados seguem as normas contidas na TAPPI (Technical Association of Pulpand Paper Industry), de acordo com a Tabela 2.

Tabela 2 - Métodos de análise da polpa

\begin{tabular}{cc}
\hline Parâmetro Utilizado & Metodologia \\
\hline Confecção de Folhas & TAPPI - 205 sp 95 \\
Número Kappa & TAPPI - 236 om 99 \\
Viscosidade & TAPPI - 230 om 99 \\
Alvura & TAPPI - 525 om 92 \\
Reversão de Alvura & TAPPI - UM 200 \\
\hline
\end{tabular}

Fonte: Autores (2020) 


\section{RESULTADOS E DISCUSSÃO}

\subsection{Alvura}

Foi estudado com o auxílio de diferentes sequências de branqueamento o efeito da extração alcalina após a hidrólise ácida, buscando o maior aproveitamento do estágio de dióxido de cloro, aumentando a reatividade da lignina antes do mesmo.

$\mathrm{Na}$ realização das sequências de branqueamento buscou-se a alvura final de $90,0 \pm 0,5 \%$ ISO. Utilizando como referência a sequência $O D_{0}(E+P) D_{1}$, cuja alvura final foi de $90,0 \%$ ISO e reversão de alvura de 3,4\% ISO.

Cada sequência foi realizada três vezes e caracterizada quanto à alvura, reversão de alvura e número Kappa. A Tabela 3 apresenta os valores médios de alvura medida até o estágio de extração alcalina com peróxido nas sequências de branqueamento realizadas, onde foi feito o teste Tukey em nível de 5\% de significância.

Tabela 3 - Valores médios de alvura obtidos nas sequências estudadas

\begin{tabular}{lcccccc}
\hline \multirow{2}{*}{ Sequência } & \multicolumn{7}{c}{ Alvuras, \%ISO } \\
\cline { 2 - 7 } & $\mathbf{A}_{\mathrm{HT}}$ & $\mathbf{A}_{\mathrm{HT}} / \mathbf{E}$ & $\mathbf{E}$ & $\mathbf{D}_{\mathbf{0}}$ & $\mathbf{( E + P )}$ & $\mathbf{D}_{\mathbf{1}}$ \\
\hline $\mathrm{A}_{\mathrm{HT}} \mathrm{D}_{0}(\mathrm{E}+\mathrm{P}) \mathrm{D}_{1}$ & 60,6 & - & - & 71,8 & $81,6 \mathrm{~A}$ & 90,1 \\
$\mathrm{~A}_{\mathrm{HT}} / \mathrm{ED}_{0}(\mathrm{E}+\mathrm{P}) \mathrm{D}_{1}$ & - & 57,4 & - & 72,6 & $82,9 \mathrm{~A}$ & 90,5 \\
$\mathrm{~A}_{\mathrm{HT}} \mathrm{ED}_{0}(\mathrm{E}+\mathrm{P}) \mathrm{D}_{1}$ & 60,6 & - & 58,3 & 72,8 & $82,6 \mathrm{~A}$ & 90,3 \\
\hline
\end{tabular}

Fonte: Autores (2020)

Em que: $A_{H T}=$ hidrólise ácida em alta temperatura; $D_{0}=$ primeiro estágio de dioxidação; $A_{H T} / E=$ estágio de hidrólise ácida em alta temperatura seguida por uma extração simples sem lavagem intermediária; $E$ = estágio de extração alcalina simples; $(E+P)$ = estágio de extração alcalina com peróxido de hidrogênio; $\mathrm{D}_{1}=$ segundo estágio de dioxidação. As letras maiúsculas comparam as linhas da coluna $(E+P)$. Médias seguidas pela mesma letra nas colunas não diferem entre si em nível de $5 \%$ de significância pelo teste Tukey.

A interpretação dos resultados obtidos do teste Tukey em nível de $5 \%$ de significância indica que as alvuras obtidas nas sequências com extração alcalina simples, com e sem lavagem, não diferem com a sequência $A_{H T} D_{0}(E+P) D_{1}$. Entretanto, esse resultado foi esperado, podendo ser justificado pelo reduzido número de 
repetições e um amplo intervalo de confiança nos resultados. Todavia, ponderando de forma minuciosa a média da alvura obtida nas sequências no estágio ( $E+P)$ observase que a sequência $A_{H T} / E D_{0}(E+P) D_{1}$ se destacou das demais, apresentando a maior alvura, enquanto que a sequência $A_{H T} E D_{0}(E+P) D_{1}$ apresentou a segunda maior alvura com $1 \%$ ISO a mais que a sequência de referência $A_{H T} D_{0}(E+P) D_{1}$. Segundo Dence e Reeve (1996) e Shackford et al. (2009), a inclusão de um estágio de extração alcalina, após o tratamento de hidrólise ácida, resulta em um aumento de alvura final da polpa.

Como mencionado por Dence e Reeve (1996), a reação mais importante do hidróxido de sódio com a lignina residual no estágio de extração alcalina é a conversão de fragmentos de ácido parcialmente degradado nas suas formas aniônicas correspondentes, o principal efeito em tais reações é um subsequente aumento na solubilidade dos fragmentos de lignina aos quais os grupos ácidos são anexados, o que nos estágios posteriores do branqueamento se torna um benefício para a remoção da lignina.

Com destaque para a sequência com extração alcalina sem lavagem, que resultou em 1,3\% ISO a mais na alvura, se comparada com a sequência $A_{H T} D_{0}(E+P)$ $\mathrm{D}_{1}$, produzindo uma avaliação positiva dessa extração sem lavagem. Considerando que apesar de não ser estatisticamente significante essa diferença em nível de 5\% de significância, na indústria, um aumento da alvura final da polpa é muito relevante, pois pequenos pontos a mais na alvura podem significar redução de gastos principalmente com consumo de químicos de branqueamento e demais custos operacionais.

É interessante considerar, portanto, que nas sequências com extração alcalina simples o consumo de dióxido de cloro foi sujeito a uma considerável redução, uma vez que na sequência $A_{H T} D_{0}(E+P) D_{1}$ foi necessária uma maior quantidade de dióxido de cloro, sendo aplicado 22,05 kg/t de $\mathrm{ClO}_{2}$ como $\mathrm{Cl}_{2}$, e para alcançar a alvura final desejada, foram necessários 21,60 kg/t e 21,75 kg/t nas sequências $A_{H T} / E D_{0}(E+P) D_{1}$ e $A_{H T} E D_{0}(E+P) D_{1}$, respectivamente. Por consequência, a adição de uma extração alcalina simples posterior à hidrólise ácida se mostra uma opção atraente, pois como afirmado 
por Colodette e Gomes (2015), nos últimos tempos as indústrias de papel e celulose têm buscado cada vez mais alternativas no branqueamento da polpa celulósica que proporcionem menores custos operacionais nos processos. E a redução da carga aplicada de dióxido de cloro durante o branqueamento, proporcionado pela adição do estágio de extração alcalina simples na sequência é uma alternativa que deve ser estudada e considerada no planejamento de redução de custos.

Tendo como referência a sequência sem o estágio de hidrólise ácida à alta temperatura $\mathrm{D}_{0}(\mathrm{E}+\mathrm{P}) \mathrm{D}_{1}$, foi possível perceber que os resultados médios de alvura foram menores, quando comparados com as sequências que utilizaram o estágio hidrólise ácida. A medição de alvura foi analisada após o estágio de extração alcalina com peróxido, sendo essa redução da alvura comum nesse ponto. E após o estágio com dióxido de cloro ocorre um aumento na alvura final da sequência.

A sequência referência $D_{0}(E+P) D_{1}$ consumiu um total de $26 \mathrm{~kg} / \mathrm{t}$ de $\mathrm{ClO}_{2}$ como $\mathrm{Cl}_{2}$. Um valor significativamente maior do que a sequência $\mathrm{A}_{\mathrm{HT}} \mathrm{D}_{0}(\mathrm{E}+\mathrm{P}) \mathrm{D}_{1}$, que consumiu 22,05 kg/t. Essa diferença representa uma redução de 15,2\% no consumo de dióxido de cloro diante da inclusão de um estágio de hidrólise ácida antes do primeiro estágio com dióxido na sequência de branqueamento, resultados semelhantes foram observados por Eiras (2002) e Ventorim et al. (2009).

Os benefícios mencionados na redução de consumo de dióxido de cloro são destacados por Colodette e Gomes (2015), estando relacionados diretamente à remoção dos ácidos hexenurônicos, que no processo de branqueamento tendem a reagir com espécies ativas derivadas do dióxido de cloro, e consequentemente aumentar o consumo desse reagente, em conformidade com os indícios dos resultados obtidos neste trabalho. À vista disso, proporcionam uma gama de vantagens com a hidrólise ácida em alta temperatura referente à sua eficiência quanto à remoção de ácidos hexenurônicos, como mencionado por Ratnieks et al. (1997), nesse aspecto está inclusa a redução no número Kappa, ao longo da sequência de branqueamento, e o aumento na resistência da polpa à reversão de alvura (VENTORIM, 2004). 


\subsection{Reversão de alvura}

Em relação à reversão de alvura que foi medida ao final das sequências de branqueamento, os dados obtidos nas análises estão disponíveis na Tabela 4.

Tabela 4 - Média da reversão de alvura obtidas nas sequências estudadas

\begin{tabular}{lcc}
\hline \multicolumn{1}{c}{ Sequências } & Alvura, \%ISO & Reversão de Alvura, \%ISO \\
\hline$A_{H T} D_{0}(E+P) D_{1}$ & $90,1 \mathrm{~A}$ & $3,0 \mathrm{~A}$ \\
$\mathrm{~A}_{\mathrm{HT}} / E D_{0}(E+P) D_{1}$ & $90,5 \mathrm{~A}$ & $2,8 \mathrm{~A}$ \\
$\mathrm{~A}_{\mathrm{HT}} \mathrm{ED}_{0}(\mathrm{E}+\mathrm{P}) \mathrm{D}_{1}$ & $90,3 \mathrm{~A}$ & $2,9 \mathrm{~A}$ \\
\hline
\end{tabular}

Fonte: Autores (2020)

Em que: $A_{H T}=$ hidrólise ácida em alta temperatura; $D_{0}=$ primeiro estágio de dioxidação; $A_{H T} / E$ = estágio de hidrólise ácida em alta temperatura seguida por uma extração simples sem lavagem intermediária; $\mathrm{E}$ = estágio de extração alcalina simples; $(\mathrm{E}+\mathrm{P})$ = estágio de extração alcalina com peróxido de hidrogênio; $\mathrm{D}_{1}=$ segundo estágio de dioxidação. As letras maiúsculas comparam as linhas da coluna "Reversão de Alvura". Médias seguidas pela mesma letra nas colunas não diferem entre si em nível de 5\% de significância pelo teste Tukey.

Analisando os resultados contidos na Tabela 4, pode-se perceber que apesar das médias de reversão de alvura nas sequências com um estágio de extração alcalina antes do $D_{0}$ se mostraram menores que na sequência sem a extração $A_{H T} D_{0}(E+P) D_{1}$, o teste Tukey realizado revelou que não há uma diferença em nível de 5\% de significância na reversão de alvura, entre as sequências com a adição de uma extração alcalina após o estágio ácido à alta temperatura.

Contudo, é importante considerar que na indústria pequenos pontos de diferença na reversão de alvura se mostram uma opção interessante, ainda mais levando em conta que a alvura final nessas sequências com extração se mostraram maiores, e o consumo de dióxido nas mesmas foram menores, permitindo ainda alcançar efeitos positivos de reversão de alvura.

Segundo Oliveira et al. (2006) e Colodette e Gomes (2015), o processo de branqueamento é um dos fatores que mais influência na estabilidade da alvura, sendo que tratamentos alcalinos são muito eficientes na remoção de extrativos e lignina residual, diminuindo assim o efeito negativo dos mesmos na reversão de alvura. 
Ao comparar as sequências $A_{H T} D_{0}(E+P) D_{1}$ e $D_{0}(E+P) D_{1}$, entende-se que, apesar da análise com o teste Tukey não mostrar diferença estatística entre elas, as médias de reversão de alvura encontradas indicam que a introdução do estágio ácido na sequência se mostra favorável com $0,4 \%$ ISO a menos na reversão de alvura para a sequência com hidrólise ácida, resultados também observados por Eiras, Colodette e Silva (2009), Forsström, Hellström e Greschik (2007) e Ventorim et al. (2009).

Segundo Eiras, Colodette e Silva (2009), polpas com um estágio de hidrólise ácida são menos propensas à reversão de alvura, devido ao seu menor teor de ácidos hexenurônicos, e cloro residual, em virtude da redução no consumo de dióxido de cloro que esse estágio proporciona. Sendo que, do ponto de vista ambiental, a hidrólise ácida pode fornecer um menor teor de organoclorados, como relatado por Gomes (2006) e Ventorim (2004), atendendo a um grande nicho do mercado.

\subsection{Número Kappa}

As análises de número Kappa foram realizadas nas polpas após os estágios de hidrólise ácida $\left(A_{H T}\right)$, hidrólise ácida seguida de extração alcalina sem lavagem $\left(A_{H T} / E\right)$, hidrólise ácida seguida de extração alcalina com lavagem $\left(\mathrm{A}_{\mathrm{HT}} \mathrm{E}\right)$ e extração alcalina com peróxido $(E+P)$.

O número Kappa é uma análise em que se determina a quantidade de lignina residual, extrativos e quaisquer substâncias que consomem permanganato de potássio (substâncias cromóforas e leucocromóforas).

Os resultados obtidos encontram-se na Tabela 5, sendo que o número Kappa depois da deslignificação com oxigênio é de 8,5 para a polpa usada na realização de todas as sequências de branqueamento deste trabalho.

Numa análise rigorosa dos dados apresentados na Tabela 5, identifica-se que ocorreu uma queda de 3 pontos na média do número Kappa do estágio $A_{H T}$ para o estágio $(E+P)$ na sequência $A_{H T} / E D_{0}(E+P) D_{1}$, constituindo o melhor resultado encontrado de número Kappa, com um diferença menor de 0,3\%ISO se comparada à sequência 
sem extração alcalina, e 0,2\%ISO em comparação com a sequência com extração alcalina com lavagem.

Tabela 5 - Média dos números Kappa obtidos nas sequências estudadas

\begin{tabular}{ccccc}
\hline \multirow{2}{*}{ Sequência } & \multicolumn{5}{c}{ Estágios } \\
\cline { 2 - 5 } & $\mathrm{A}_{\mathrm{HT}}$ & $\mathrm{A}_{\mathrm{HT}} / \mathrm{E}$ & $\mathrm{E}$ & $(\mathrm{E}+\mathrm{P})$ \\
\hline $\mathrm{OA}_{\mathrm{HT}} \mathrm{D}_{0}(\mathrm{E}+\mathrm{P}) \mathrm{D}_{1}$ & 6,7 & - & - & $3,7 \mathrm{~A}$ \\
$\mathrm{OA}_{\mathrm{HT}} / \mathrm{ED}_{0}(\mathrm{E}+\mathrm{P}) \mathrm{D}_{1}$ & - & 6,4 & - & $3,4 \mathrm{~A}$ \\
$\mathrm{OA}_{\mathrm{HT}} \mathrm{ED}_{0}(\mathrm{E}+\mathrm{P}) \mathrm{D}_{1}$ & 6,7 & - & 6,5 & $3,6 \mathrm{~A}$ \\
\hline
\end{tabular}

Fonte: Autores (2020)

Em que: $A_{H T}=$ hidrólise ácida em alta temperatura; $D_{0}=$ primeiro estágio de dioxidação; $A_{H T} / E=$ estágio de hidrólise ácida em alta temperatura seguida por uma extração simples sem lavagem intermediária; $E$ = estágio de extração alcalina simples; $(E+P)=$ estágio de extração alcalina com peróxido de hidrogênio; $D_{1}=$ segundo estágio de dioxidação. As letras maiúsculas comparam as linhas da coluna $(E+P)$. Médias seguidas pela mesma letra nas colunas não diferem entre si em nível de 5\% de significância pelo teste Tukey.

As duas sequências com estágio de extração alcalina simples adicional se mostraram mais vantajosas na redução do número Kappa, evidenciando o efeito positivo do estágio na diminuição dessa grandeza. Os resultados obtidos neste estudo estão em conformidade com Dence e Reeve (1996), que afirmam que o aumento da alcalinidade antes do estágio com dióxido de cloro aumenta o potencial de remoção da lignina.

Ainda que o teste Tukey realizado em nível de 5\% de significância não aponte que há diferença estatística entre as sequências, como descrito na Tabela 5, quando se avalia o efeito do número Kappa na indústria de papel e celulose, a diminuição identificada na média dos resultados se mostra muito importante.

\section{CONCLUSÕES}

A introdução de um estágio de extração alcalina simples na sequência de branqueamento após a hidrólise ácida e anterior ao primeiro estágio com dióxido de cloro teve um efeito positivo sobre as variáveis analisadas no presente trabalho, 
indicando um aumento da reatividade da lignina numa fase muito importante no branqueamento, sendo observada assim uma ligeira redução no consumo de dióxido de cloro, com o destaque para a sequência $A_{H T} / E D_{0}(E+P) D_{1}$.

O número Kappa se revelou menor, com a inclusão da extração alcalina simples, se comparado com as sequências de branqueamento estudadas nesse projeto sem essa extração.

Também foi observada uma redução na reversão de alvura para as sequências com extração alcalina com e sem lavagem.

A respeito do estudo do estágio ácido à alta temperatura, foi constatado na comparação da sequência $A_{H T} D_{0}(E+P) D_{1}$ com a sequência referência $D_{0}(E+P) D_{1}$, que a hidrólise ácida realizada nas condições deste trabalho apresenta inúmeras vantagens industriais que foram desde o alcance de alvuras finais mais altas com menor consumo de dióxido de cloro até diminuição da reversão de alvura e número Kappa.

\section{AGRADECIMENTOS}

Os autores agradecem o suporte financeiro oferecido pela FAPESP (Fundação de amparo à pesquisa do estado de São Paulo).

\section{REFERÊNCIAS}

CADENA, E. M.; VIDAL, T.; TORRES, A. L. Can the laccase mediator system affect the chemical and refining properties of the eucalyptus pulp?. Bioresource Technology, New York, v. 101, n. 21, p. 3554 - 3560, nov. 2010.

CADENA, E. M. et al. On hexenuronic acid (HexA) removal and mediator coupling to pulp fiber in the laccase/mediator treatment. Bioresource Technology, New York, v. 102, n. 4, p. 3911-3917, feb. 2011.

COLODETTE, J. L.; GOMES, V. J. Branqueamento de polpas químicas para papel: estágio ácido para remoção de ácidos hexenurônicos. In: COLODETTE, J. L.; GOMES, F. J. B. (ed.). Branqueamento de polpa celulósica: da produção da polpa marrom ao produto acabado. Viçosa, MG: Editora UFV, 2015. seç. V, cap. 2, p. 313-352.

DENCE, C. W.; REEVE, D. W. Pulp bleaching: principles and practice. Atlanta: Tappi Press, 1996. 
EIRAS, K. M. M. Otimização do estágio de branqueamento de polpa kraft de eucalipto com dióxido de cloro em alta temperatura. 2002. Dissertação (Mestrado em Ciência Florestal) Universidade Federal de Viçosa, Viçosa, MG, 2002.

EIRAS, K. M. M.; COLODETTE, J. L.; SILVA, V. L. The role of bound chlorine in the brightness reversion of bleached hardwood kraft pulp. Química Nova, São Paulo, v. 32, n. 1, p. 51-55, set. 2009.

FORSSTRÖM, A.; HELLSTRÖM, P.; GRESCHIK, T. Ageing of eucalyptus pulps: effects on brightness and viscosity. In: INTERNATIONAL COLLOQUIUM ON EUCALYPTUS KRAFT PULPS, ORAL SESSIONS, 3., 2007, Bohus. Proceedings [...]. Bohus: EkaChemicals AB, 2007. p. 1-7.

GOMES, C. M. Tratabilidade dos efluentes de branqueamento de polpa kraft de eucalipto com tecnologias de hidrólise ácida/dióxido de cloro à quente. 2006. Dissertação (Mestrado em Ciência Florestal) - Universidade Federal de Viçosa, Viçosa, MG, 2006.

OLIVEIRA, R. L. et al. The effect of wood supply and bleaching process on pulp brightness stability. Revista Árvore, Viçosa, MG, v. 30, n. 3, p. 439-450, mar. 2006.

RATNIEKS, E. et al. Improved Eucalyptus pulp bleachability via high temperature acid treatment. In: INTERNATIONAL EMERGING TECHNOLOGIES CONFERENCE AND EXHIBITION, 1997, Orlando. Proceedings [...]. Orlando: [s. n.], 1997, p. 91-98.

REIS, H. M. Processos de branqueamento e recuperação química. In: SENAI-SP. Celulose. São Paulo: SENAI, 2013.

SHACKFORD, L. D. et al. Optimizing alcaline extraction kraft pulp bleaching. Tappi Journal, Atlanta, v. 8, n. 1, p. 12-19, 2009.

TECHNICAL ASSOCIATION OF THE PULP AND PAPER INDUSTRY. Testing procedures of Technical Association of the Pulp and Paper Industry. In: TECHNICAL ASSOCIATION OF THE PULP AND PAPER INDUSTRY. Standard Methods. Atlanta: TAPPI Press, 2001. 1 CD-ROM.

VENTORIM, G. Estudo das reações da lignina e dos ácidos hexenurônicos em polpa kraft de eucalipto com oxigênio, dióxido de cloro, ácido sulfúrico e ozônio. 2004. Tese (Doutorado em Ciência Florestal) - Universidade Federal de Viçosa, Viçosa, MG, 2004.

VENTORIM, G. et al. A influência dos ácidos hexenurônicos no rendimento e na branqueabilidade da polpa kraft. Química Nova, São Paulo, v. 32, n. 2, p. 373-377, fev. 2009.

VENTORIM, G.;COLODETTE,J.L.; EIRAS, K. M.O destino deespécies decloro durante o branqueamento com dióxido de cloro a altas temperaturas. O Papel, São Paulo, v. 70, n. 8, p. 39-50, ago. 2009.

VUORINEN, T. et al. Selective hydrolysis of hexenuronic acid groups and its application in ECF and TCF bleaching of kraft pulps. Journal of Pulp and Paper Science, Montreal, v. 25, n. 5, p. 155-162, 1999.

ZHANG, $H$. et al. Removal of hexenuronic acid to reduce AOX formation in hot chlorine dioxide bleaching of bagasse pulp. Industrial Crops and Products, Amsterdam, v. 128, p. 338-345, feb. 2019. 


\section{Contribuição de Autoria}

\section{1 - Iliane Rodrigues de Oliveira}

Engenheira Industrial Madeireira, Bacharel

https://orcid.org/0000-0001-9066-5783•iliane.rodrigues@unesp.br

Contribuição: Conceituação, Curadoria de dados, Análise Formal, Investigação, Metodologia, Administração do projeto, Escrita - revisão e edição, Visualização, Software, Obtenção de financiamento

\section{2 - Gustavo Ventorim}

Bacharel em Química, Dr., Professor

https://orcid.org/0000-0001-9238-4141·gustavo.ventorim@unesp.br

Contribuição: Conceituação, Obtenção de financiamento, Investigação, Metodologia, Administração do projeto, Recursos, Supervisão, Validação e Escrita - revisão e edição

\section{3 - Ana Paula Almeida Ferraz}

Engenheira Industrial Madeireira, Ma., Professora https://orcid.org/0000-0001-7752-7028•ap.ferraz@unesp.br

Contribuição: Escrita - primeira redação, Escrita - revisão e edição

\section{Como citar este artigo}

Oliveira, I. R.; Ventorim, G.; Ferraz, A. P. A. Efeito da extração alcalina na redução do número Kappa no branqueamento ECF. Ciência Florestal, Santa Maria, v. 31, n. 1, p. 157-170, 2021. DOI 10.5902/198050344. Disponível em: https://doi.org/10.5902/198050344. Acesso em: xx mês abreviado $202 x$. 